Cahiers d'études italiennes

$7 \mid 2008$

NOVECENTO... E DINTORNI

Images littéraires de la société contemporaine (3)

\title{
La sorcière comme image de la différence dans $L a$ chimera de Vassalli
}

Stefano Magni

\section{OpenEdition}

Journals

Édition électronique

URL : http://journals.openedition.org/cei/910

DOI : $10.4000 /$ cei.910

ISSN : 2260-779X

\section{Éditeur}

UGA Éditions/Université Grenoble Alpes

\section{Édition imprimée}

Date de publication : 15 mai 2008

Pagination : 53-63

ISBN : 978-2-84310-121-2

ISSN : $1770-9571$

\section{Référence électronique}

Stefano Magni, «La sorcière comme image de la différence dans La chimera de Vassalli », Cahiers d'études italiennes [En ligne], 7 | 2008, mis en ligne le 15 novembre 2009, consulté le 01 mai 2019. URL : http://journals.openedition.org/cei/910 ; DOI : 10.4000/cei.910 


\title{
LA SORCIÈRE COMME IMAGE DE LA DIFFÉRENCE DANS LA CHIMERA DE VASSALLI
}

\author{
Stefano Magni \\ Université Stendhal-Grenoble 3, GerCI
}

Vassalli publie en 1990 son roman le plus connu: La chimera $^{1^{*}}$. L'histoire, qui se déroule au XVII ${ }^{e}$ siècle, met en scène la vie assez brève d'Antonia, jeune fille accusée d'être une sorcière et par conséquent poursuivie et brûlée par le Tribunal de l'Inquisition. Notre intention est de découvrir quel est le lien entre le destin de sorcière et le sujet de la différence. Nous montrerons ainsi comment la diversité domine la scène du roman de Vassalli et essaierons de découvrir l'idéologie de l'auteur par rapport à ce sujet. Pour ce faire, nous mettrons le roman en relation avec son modèle, I promessi sposi, en montrant le parallélisme qui lie les personnages des deux textes et en expliquant, enfin, comment l'histoire de Vassalli ré-interprète son modèle.

\section{Le personnage d'Antonia}

Antonia est une orpheline qui grandit dans «La casa di Carità » de Novare. Vers l'âge de dix ans, elle est adoptée par un généreux couple de paysans "della Bassa", Bartolo et Francesca. Elle quitte alors l'orphelinat et va vivre dans le petit village de Zardino. Mais, dans la nouvelle réalité, la jeune fille est l'étrangère et autour de ce point se joue son destin, car la société restreinte est méfiante envers tout ce qui vient de l'extérieur. Dans La chimera, ce comportement sociologique devient intolérance.

Elle est de plus, en tant qu'enfant abandonnée, la conséquence d'un péché charnel, et à cause de cela, dès sa naissance, est touchée par le démon. Pour considérer la jeune fille comme une sorcière, il ne faut donc pas attendre les comportements anomaux et libres qu'elle aura quand elle sera adolescente, son statut d'orpheline étant suffisant. Dans l'esprit des

\footnotetext{
* Notes p. 62. 
commères, une relation s'instaure d'emblée entre la personne étrangère, la fille du péché charnel, et la sorcière. Elles expriment tout de suite leur jugement moral: "Anche questa ci doveva capitare! Un'esposta a Zardino! In mezzo ai nostri figli! [...] Una figlia del Diavolo! Una piccola stria!» (p. 47).

La présence d'Antonia est hors commun et cet aspect déstabilise les gens. Le village pourrait encore comprendre l'adoption d'un garçon qui se chargerait des travaux à la campagne, mais ne conçoit pas le choix d'une fille:

$\mathrm{Ma}$ andare a prendere una femmina, in città, era una cosa che non stava né in cielo né in terra, che non s'era mai vista. Un controsenso! "Come cambiano i tempi - commentavano. - E pensare che le nostre madri, e le nostre nonne, affogavano le figlie nella Crosa (cioè: nella roggia del mulino) il giorno stesso che nascevano, se erano troppe, o se non avevano più il latte per allattarle, o se le annate erano scarse".

(p. 46-47) ${ }^{2}$

Avec le choix d'un amour clandestin, Antonia accélère sa chute. Elle tombe amoureuse d'un "camminante», un vagabond qui n'est pas intégré non plus à la vie communautaire et qu'elle rencontre en secret. Selon le village, la seule relation légitime est celle du mariage qui a pour but la constitution d'un nouveau foyer fondé dans le réseau des relations codifiées. Le rapport fautif des deux jeunes sera donc forcément puni.

En nous référant au grand roman italien qui se déroule au XVII siècle, I promessi sposi ${ }^{3}$, nous pouvons constater que toute l'histoire d'Antonia est le renversement du destin de Lucia ${ }^{4}$. Il est facile de remarquer que Lucia est née dans le village où elle habite dans le respect des règles, alors qu'Antonia est une orpheline étrangère. A cet aspect correspond le fait que l'histoire de Renzo et Lucia a "un lieto fine», alors qu'Antonia meurt condamnée. La relation entre Antonia et Lucia est fondée sur l'opposition. Lucia respecte les canons prévus par la petite société du village, en suivant le modèle de la jeune fille honnête, dévouée, et prête au mariage, Antonia bouleverse toutes ces caractéristiques: aux regards baissés, aux hésitations et aux pudeurs de Lucia, Antonia réplique, par exemple, par des phrases audacieuses qui la conduisent à son atroce destin.

La critique a d'ailleurs mis en rapport La chimera avec I promessi sposi, à raison, car un rapport incontournable, au niveau de la géographie, de la chronologie et des personnages existe entre les deux histoires, qui présentent aussi des analogies textuelles, voire des intertextualités. Les deux romans ont, de plus, une préface selon laquelle l'écrivain s'est limité à adapter un manuscrit ignoré auparavant. 
Or, l'histoire de Vassalli parle d'une sorcière, alors que Manzoni glisse sur le sujet, en lui accordant peu d'attention. En 1823, l'écrivain nie d'ailleurs toute valeur morale et littéraire au "guazzabuglio di streghe [e] spettri ${ }^{5}$ " et considère la sorcellerie comme extravagante et contraire à la vérité et à la raison. Mais son opinion ne rend pas compte de l'amplitude du phénomène au XVII siècle, période qui est aussi synonyme de chasse aux sorcières.

Manzoni abordera ce thème dans la Storia della colonna Infame (1842), l'œuvre qui suit les Promessi sposi et qui s'occupe de la condamnation des "untori», c'est-à-dire ceux qui, selon les dires du peuple, furent responsables de la peste à Milan en peignant les cadres des portes d'un onguent spécial $^{6}$. Le roman de Vassalli remplit alors un vide qui rend, par là, honneur à toute la culture orale submergée de ce siècle, car le livre dénonce la répression de la diversité, et relit sous une autre optique le roman fondateur de la littérature italienne.

Comme la critique l'a souvent remarqué, si Manzoni regarde d'un œil très fin et reproduit attentivement la société du XVII ${ }^{e}$ siècle ${ }^{7}$, ses représentations de la vie rurale sont souvent cachées derrière le voile d'une vision idyllique, fondée sur le stéréotype, dont l'exemple le plus archétypique est le fameux passage de l'adieu aux monts que les fiancés fugitifs adressent à leur terre: "Addio, monti sorgenti dall' acque ed elevati al cielo $[\ldots]^{8}$ ".

Vassalli, en revanche, décrit un microcosme vif, riche en fêtes, en traditions, et en hérésies. C'est un lieu qui accepte la coprésence des contraires: sacré et profane, rigorisme et libertinage, espace clos et ouvert. Il s'agit d'un monde païen, enseveli par l'Église catholique et par la "modernisation» de la Contre-réforme ${ }^{9}:$ La chimera est ainsi une mémoire des diversités homologuées par l'Église romaine. L'un des messages les plus forts du roman nous transmet l'idée que la Contre-réforme a effacé une richesse culturelle unique, en tranchant avec notre tradition.

La campagne de Vassalli comporte donc deux aspects. Elle accueille les paysans du village, enfermés dans leurs petites disputes, dans leur respect de l'ordre constitué ou dans leurs peurs. Mais elle accueille aussi un monde parallèle, illégal et caché, un univers souvent oublié, que Vassalli représente avec une extraordinaire vivacité. Il s'agit des « risaroli », les travailleurs saisonniers qui descendaient des villages de montagne; des "madonnari», les artistes de province qui erraient avec leurs outils; des "camminanti", sorte de vagabonds qui vivaient dans l'indiscipline, dans les tricheries et dans les violences; des "esposti», les nouveaux-nés laissés à l'entrée des orphelinats; et, finalement, des sorcières. 
Dans le roman de Vassalli, toute cette diversité oubliée devient une valeur, et l'image de campagne, lieu de fêtes, rites anciens, croyances païennes, semble être inspirée, pour certains aspects, par des textes historiographiques consacrés au sujet. Une référence privilégiée est ici l'œuvre de Carlo Ginzburg (Storia notturna ${ }^{10}$, Il formaggio e $i$ vermi ${ }^{11}$ et $I$ benandanti $^{12}$ ), dont l'influence sur Vassalli a été, à notre avis, souvent négligée et qui revient, d'après nous, dans les mots hérétiques d'Antonia.

Vassalli est en fait très attentif à signaler l'existence de rites qui ont dû se modifier au cours des siècles, suivant les préceptes de la religion. Il mentionne des traditions qui ont évolué, comme le culte des «madri» (p. 86), à savoir, dans le mythe, des matrones celtiques de la fertilité, dont le culte s'est poursuivi, mélangé au christianisme, pendant des siècles.

Nous trouvons aussi d'autres liens possibles entre les deux auteurs. Les "Tempora» de Ginzburg sont citées par Vassalli (p. 187) ; les fêtes populaires, "balli, carnevali, feste di maggio, feste del raccolto» (p. 187) que don Teresio veut effacer, sont souvent abordées dans les textes de Ginzburg. Comme Ginzburg, Vassalli observe donc les changements qui conduisent les traditions païennes à se mêler aux rites catholiques. C'est au XVII siècle que la force des bacchanales, réprimée par les nouvelles coutumes établies à Trento, éclate dans deux directions opposées. D'un côté, elle choisit la solution interdite et orgiastique des sabbats où, selon les dires, le démon aussi se joindrait aux participants. D'un autre côté, cette énergie refoulée éclate légalement dans la répression de ces mêmes comportements: l'exécution des sorcières donne lieu à des comportements libératoires et violents. Lorsqu'elle est menée au sacrifice, Antonia subit des attaques barbares et farouches de la part de la foule. Ces agressions sont une vengeance contre celle qui a porté le malheur, mais aussi, plus simplement, les phases d'un rite collectif. L'Église et le peuple qui ont été en désaccord pendant tout le roman - le symbole de leur incompréhension étant le rapport contrasté entre don Teresio et le village de Zardino rejoignent à travers cette pratique un nouvel accord.

Au moment de l'exécution finale, les deux parties se trouvent sur le même front, le supplice d'Antonia étant la conjonction légale du rigorisme catholique et de l'esprit libératoire païen. La sorcière se trouve finalement au delà de la culture populaire et religieuse, et le fait qu'elle soit étrangère facilite cette conjonction. 


\section{Pourquoi l'histoire d'une sorcière}

Ces réflexions nous semblent importantes, car elles nous conduisent à réfléchir sur la signification que peut avoir aujourd'hui l'histoire romanesque d'une sorcière du XVII siècle: La chimera est une réponse à l'idéologie manzonienne, mais en quoi ces valeurs concernent-elles l'idée de différence?

La valeur la plus importante de l'hypotexte est la "provvidenza», soit l'idée d'un dessein divin qui règle le déroulement de la vie de chacun. La critique la plus récente tend à réduire l'importance de la valeur religieuse et de la providence dans I promessi sposi $i^{13}$, mais on a longtemps écrit et répété que tout le roman gravite autour de cette idée qui est aussi une synthèse de l'idéologie manzonienne, «il sugo di tutta la storia», et Vassalli nous semble surtout lire le roman selon cette perspective traditionnelle.

On peut se demander comment la foi manzonienne s'articule avec la méfiance envers l'institution religieuse et avec le nihilisme de La chimera. Nous essayerons de répondre au niveau du macro-texte et du micro-texte en commençant par ce deuxième. Nous avons en effet d'abord cherché des récurrences du mot "Provvidenza» dans le roman du XXe siècle et nous n'avons trouvé qu'une seule utilisation du terme, dont le contexte peut nous aider à comprendre la position de Vassalli par rapport à l'idée qu'il véhicule. L'épisode se trouve au début du roman, lorsqu'Antonia est encore à l'orphelinat. Sa camarade Rosalina, plus âgée, et qui a connu le monde en se prostituant, prend un rôle d'éducatrice: sur un ton cynique, elle renverse les valeurs manzoniennes et sous-entend que personne ne peut aider une orpheline lors de sa rencontre avec le monde. Son seul soutien réside dans son corps. Le message est très dur, car la «Provvidenza» se trouve parmi les jambes des jeunes filles: "la sola cosa che vi aiuterà ad affrontare il mondo è quell'affare che avete tra le gambe. Lì c'è la Provvidenza, quella vera, l'unica che ci viene in aiuto anche quando il mondo intero ci è contro!» (p. 34).

Qu'en est-il de la providence au niveau du macro-texte? Nous savons que Manzoni applique sa vision du monde à l'histoire de Renzo et Lucia: ils ont la foi, ne perdent pas confiance, et traversent toutes les difficultés en parvenant à un joyeux mariage ${ }^{14}$. Dans I promessi sposi, personnages et événements suivent une direction "prédestinée", mais qui correspond à leur bien. Antonia, en revanche, est conduite au bûcher qui remplace la fête de son mariage. On peut alors se demander quelle est la valeur vassallienne qui succède à la providence manzonienne. 
Chez le parodiste tout semble conduire vers le nihilisme. Si aux indécisions de Lucia correspondent des énergies universelles qui déplacent les pions sur l'échiquier en sa faveur, les hésitations d'Antonia se heurtent au néant: aucune intervention ni aucune loi ne peut la sauver.

Comme nous essaierons de le démontrer, la différence entre les deux textes nous intéresse pour analyser le procédé mis en acte par Vassalli. Pour comprendre les raisons de ce roman et son idée de la différence, nous allons proposer un parallélisme entre les personnages des deux œuvres. Des détails communs rapprochent en effet les figures mineures des deux romans, ce qui épaule la théorie de la parodie. Des liens évidents concernent, d'abord, les personnages des religieux. Cette analyse nous servira à mieux comprendre le rapport entre les institutions et la différence dans les deux textes.

Pour l'économie du roman, la plus importante de ces figures est l'évêque Bascapé - comparable à Federigo Borromeo - que l'on peut considérer comme le deuxième protagoniste de l'histoire. Bascapé, comme son double, est un personnage historique.

Les fonctions diégétiques des deux figures sont, de plus, semblables. Premièrement, au niveau du macro-texte, car ils représentent la voix officielle de l'Église, ensuite au niveau du micro-texte, pour lequel nous renvoyons à l'article de Verina Jones qui confirme l'existence d'un rapport d'intertextualité 15 .

Les deux pasteurs sont directement opposés. Un discours tenu lors d'une homélie nous semble représentatif. Selon Manzoni, Borromeo parle d'une manière simple et touche les cœurs de tous: "anche i più duri di testa, i più ignoranti, andavan dietro al filo del discorso [di Borromeo]. Andate ora a domandar loro se saprebbero ripeter le parole che diceva: sì ; non ne ripescherebbero una ; ma il sentimento lo hanno qui» (p. 158). D’après Vassalli, Bascapé ne se fait pas comprendre, car il ne parle pas en patois, mais en italien: "in lingua». La parodie a évidemment une valeur idéologique et exprime la position de Vassalli face à l'institution religieuse: "della predica del vescovo capirono soltanto alcune parole e alcune frasi, o addirittura non capirono niente: e ciò, per il semplice motivo che il vescovo predicava in lingua, mentre i contadini parlavano in dialetto" (p. 158). Vassalli nous donne ainsi une image plus froide et distante du représentant de l'Église. Les positions idéologiques des deux écrivains divergent aussi dans d'autres cas, comme dans la représentation de don Abbondio et des correspondants don Teresio et don Michele ${ }^{16}$. Mais le cas concernant padre Cristoforo nous semble exemplaire. 
Celui-ci trouve une correspondance en Pietro Maffiolo, un laïc qui par certains aspects recouvre une même fonction diégétique. Maffiolo apparaît sur scène pour témoigner devant le Tribunal de l'Inquisition lorsque pour Antonia la situation tourne au pire. Son intervention tend à protéger la jeune fille, bien que son geste soit insuffisant. L'intercession de padre Cristoforo auprès de don Rodrigo rappelle cette scène. Les deux tentatives échouent, et dans les deux cas les protagonistes se trouvent dans une position psychologiquement inférieure par rapport à l'interlocuteur: l'inquisiteur accueille d'ailleurs Maffiolo avec des mots ironiques (p. 248), alors que don Rodrigo adresse à padre Cristoforo un sourire railleur ${ }^{17}$. Dans ce cas aussi, comme le rappelle encore une fois Verina Jones, "The points of contact between the textes are, once again, not limited to the diegetic level, but include clear verbal borrowings ${ }^{18}$ ».

Cette homologie présente aussi des points divergents. Les buts idéologiques, en particulier, different, ce qui crée l'effet de parodie. Dans I promessi sposi, c'est un représentant de l'Église qui est menacé et agressé verbalement par un noble qui transgresse l'ordre social et les lois. L'institution - dans ce cas religieuse - est donc la victime. Dans La chimera, la perspective est complètement renversée, car c'est l'institution qui attaque le citoyen.

Or nous savons que Manzoni voulait réformer l'Église catholique et qu'il la critiquait de son regard positiviste, à cause de la chasse aux sorcières, en particulier. Son esprit rationnel ne pouvait pas croire à la magie et à l'occultisme. Manzoni néglige des aspects de l'Église du XVII siècle, mais il est vrai que dans I promessi sposi sa critique n'est qu'ébauchée ${ }^{19}$. Il n'aborde pas, par exemple, le fait que Federigo Borromeo fut, de son vivant, rangé en première ligne contre la chasse aux sorcières. Vassalli, nous l'avons brièvement anticipé, peint en revanche Borromeo comme un politicien rusé et transforme ainsi l'image du noble père spirituel et met ensuite en relief l'aspect persécuteur de l'Église qui ne fut pas suivi par son modèle.

Dans le roman de Manzoni, l'organisme ecclésiastique symbolise la confiance, surtout grâce à des religieux qui interprètent et vivent l'authentique esprit chrétien ${ }^{20}$, alors que pour Vassalli le danger provient de l'institution elle-même. La différence réside précisément dans cet aspect. Si pour Vassalli le péril est porté par l'Église, celui qui sort des règles et des lois morales communes ne constitue plus un danger. Suivant cette interprétation, quelle sera, dans La chimera, la place et le rôle des seigneurs qui semaient la peur dans I promessi sposi? 
Dans La chimera, le noble voué au mal est Giovan Battista Caccia, dit Caccetta, qui est à la fois, comme le dit Vassalli lui-même, le détournement de l'Innominato et de Don Rodrigo ${ }^{21}$. Mais, comme nous allons le montrer, son image nous semble surtout une parodie de l'Innominato. L'histoire de Caccetta reprend les stéréotypes du noble maudit qui consacre sa vie au crime et à la haine. Mais, à la différence de l'Innominato, certains aspects le relèguent dans un registre comique. Son aspect physique tout d'abord est anti-héroïque: tous ceux qui le rencontrent sont stupéfaits et se demandent comment un «omiciattolo» (p. 111) pareil peut avoir semé la terreur dans la région. L'Innominato avait une lumière sinistre dans les yeux qui trahissait force d'esprit, alors que Caccetta a des yeux «sporgenti e lucidi» (p. 111) et un teint jaune.

En traçant un profil modeste du symbole du mal, Vassalli met d'autant mieux en évidence le fait que le véritable danger se situe du côté de l'institution religieuse. Il nous semble donc que le fait de minimiser la figure de Caccetta a une valeur idéologique.

On pourrait dire que ce nihilisme dérive de «certe carte». On reviendrait ainsi à délégitimer l'auteur à travers l'expédient du manuscrit. Manzoni a écrit son roman à partir de personnages historiques du XVII ${ }^{e}$ siècle, mais en inventant les figures de Renzo et de Lucia. Si Vassalli veut parodier I promessi sposi, en se situant sur le même niveau, il faut penser qu'Antonia est une création de sa fantaisie.

Toute la critique que nous avons consultée - jusqu'au livre monographique récent de Cristina $\mathrm{Nesi}^{22}$ - ignore le problème, ou bien considère Antonia comme un personnage qui a réellement existé. Ce faisant, elle reconnaît que Vassalli a vraiment tiré l'histoire de la sorcière de "certe carte». La différence nous semble substantielle, car si Antonia est un personnage inventé, le défi littéraire de la réécriture acquiert une valeur encore plus subtile.

Plusieurs données nous font d'ailleurs soupçonner ce fait: au niveau du micro-texte, par exemple, c'est lorsque Vassalli déclare avoir pris son inspiration dans «certe carte, che se fossero rimaste al loro posto ora sarebbero inaccessibili» (p. 5), que se réalise le moment parodique le plus fort de la préface manzonienne. Ce détail pourrait constituer la clef qui nous permet de lire la préface comme un détournement parodique. Nous aurions ainsi des éléments pour considérer comme inventée l'histoire d'Antonia. 


\section{La valeur du roman - la critique de notre Histoire}

Il nous paraît évident que Vassalli s'est documenté sur le phénomène des procès contre les sorcières au XVII siècle ; nous croyons cependant que l'histoire d'Antonia, comme il nous la raconte, n'a pas vraiment été puisée dans une source historiographique.

Si Vassalli avait vraiment trouvé un document attestant un procès contre une orpheline nommée Antonia, née en 1590 et immolée en 1610, nous devrions encore nous demander dans quelle mesure l'auteur l'aurait exploité pour construire son histoire. Les "certe carte» ne semblent en somme pas juridiques, car Vassalli ne sonde pas les potentialités narratives de ce type de document. De plus, si les sources de Vassalli avaient été de nature juridique, il y aurait eu des recherches au niveau des études des traditions populaires ${ }^{23}$.

On pourrait penser que la source de Vassalli est un manuscrit littéraire dont la valeur historique n'a pas été confirmée. Dans ce cas, le parallélisme avec Manzoni serait encore plus évident: comme dans l'hypotexte, le document ne serait qu'un prétexte, un jeu littéraire évident ${ }^{24}$. Il nous semble, répétons-le, que la visée de La chimera est de réécrire Les Fiancés. Vassalli semble intéressé par le fait de remettre en discussion l'idée de providence ou, plus largement, les valeurs manzoniennes, en réécrivant et en renversant son modèle.

On peut alors se demander quel sens acquiert le roman, et comment nous pouvons mettre nos argumentations en relation avec l'idée de différence. La valeur du roman réside dans le fait d'avoir porté témoignage de l'époque de la chasse aux sorcières, d'avoir ainsi comblé le manque ethnographique et sociologique des Promessi sposi, et d'avoir repéré dans le présent les mêmes mécanismes sociaux qu'hier. Manzoni parle du XIX siècle à travers le XVII ${ }^{e}$ siècle mais, pour lui, la chasse aux sorcières est un phénomène qui concerne le passé, alors que, pour Vassalli, on peut le repérer dans les mécanismes sociaux d'aujourd'hui ; c'est ce que suggère aussi, parmi d'autres éléments, la structure cyclique de son roman. L'écriture acquiert alors une valeur capitale, car elle enseigne et transmet la mémoire.

Si Manzoni nous disait que la providence aide les gens, Vassalli nous rappelle qu'il n'y a pas de providence et que la société n'accepte que ceux qu'elle considère comme normaux. Si, dans ses critiques, Manzoni avait un esprit réformateur et voulait améliorer l'Église selon une optique moderne, Vassalli semble nier, d'une façon postmoderne, tout espoir de 
progrès, en mettant en évidence le danger qui se cache dans les institutions qui ont marqué la modernité, comme l'Église catholique.

Finalement, on peut dire que les deux livres expriment un même concept, mais selon une perspective différente: si l'écrivain du XIX siècle nous montrait qu'en respectant les règles le destin nous sourit, Vassalli tire les conséquences logiques de ce raisonnement, à savoir que l'histoire et la société éliminent ou punissent ceux qui s'écartent du bon chemin.

Dans Les Fiancés, une justice supérieure punit celui qui n'accepte pas la morale commune, ou intervient pour changer sa vie (c'est ce qui arrive à l'Innominato); dans La Chimère, toute loi - et nous pourrions dire tout métarécit - ayant perdu sa crédibilité, le seul droit qui règne relève de la violence, de la barbarie, de la brutalité et de l'irrationnel. Pour éliminer les causes du mal, la société a alors besoin d'un bouc émissaire, qui est choisi sur la base de sa différence.

\section{Notes}

1. S. Vassalli, La chimera, Torino, Einaudi, 1990. Pour les citations du roman, nous donnerons directement le numéro de page.

2. Remarquons l'intervention méta-diégétique du narrateur sous forme de voix de commentaire: "(cioè: nella roggia del mulino)".

3. A. Manzoni, I promessi sposi [1840], in Tutte le opere, Milano, Mondadori, 1985.

4. De nombreuses références textuelles confirment d'ailleurs que ce parallélisme est intentionnel.

5. A. Manzoni, Tutte le opere, Milano, Mondadori, 1985, vol. V, tome III, p. 254.

6. Cf. Processo agli untori : cronaca e atti giudiziari, sous la dir. de G. Farinelli et E. Paccagnini, Milano, Garzanti, 1988. Dans la Storia della colonna infame, Manzoni se justifie aussi de ne pas avoir traité avec suffisamment d'amplitude cet épisode de sorcellerie dans Les Fiancés.

7. Voir, par ex. S. Nigro, Il secondo romanzo: i "Promessi sposi", in Manzoni [1978], Bari, Laterza, 1988, p. 162-174.

8. A. Manzoni, I promessi sposi, in op. cit., cap. VIII, p. 149.

9. Le Concile de Trente, qui décrète le rigorisme, se termine en 1564.

10. C. Ginzburg, Storia notturna: una decifrazione del sabba, Torino, Einaudi, 1989. Comme nous l'avons écrit Vassalli a avoué avoir trouvé beaucoup de matériel vers la fin de l'écriture de son roman. Nous pouvons soupçonner que parmi ses sources tardives il y avait ce texte de Ginzburg, et aussi un texte très intéressant paru toujours en 1989: G. Farinelli, E. Paccagnini, Processo per stregoneria a Caterina de Medici, 1616-1617, Milano, Rusconi, 1989.

11. C. Ginzburg, Il formaggio e i vermi: il cosmo di un mugnaio del '500, Torino, Einaudi, 1976.

12. C. Ginzburg, I benandanti: stregoneria e culti agrari tra Cinquecento e Seicento, Torino, Einaudi, 1966.

13. Nous nous référons en particulier à P. Alberti, I porcellini d'India e il pastorello : personaggi dei Promessi sposi di Manzoni: fine di un messaggio cattolico, Roma, Armando, 2001, où l'auteur contredit la vision canonique du roman «religieux » de Manzoni. A propos de la providence, lire p. 141. A. Spranzi, Il segreto di Alessandro Manzoni: che cosa nasconde l'autore dei Promessi Sposi? Una manzoniana detective story, Milano, UNICOPLI, 2001, p. 205, se situe dans le même sillage.

14. Voire une "commedia matrimoniale ", selon S. Nigro, Il secondo romanzo..., in Manzoni, op. cit., p. 173. 
15. V. R. Jones "Intertextual patterns: I promessi sposi in La chimera", in Italian Studies, Leeds, vol. XLVII, 1992, p. 62-63.

16. Don Michele, de plus, le faux prêtre qui représente les anciens cultes païens et qui est renvoyé de sa fonction, symbolise encore une forme de différence qui doit s'homologuer après le Concile de Trente.

17. A. Manzoni, I promessi sposi, in op. cit., ch. VI, p. 92.

18. Cf. V.R. Jones, "Intertextual patterns: I promessi sposi in La chimera", op. cit., p. 60.

19. La critique la plus récente tend à nuancer cette position. Comme nous le verrons aussi ensuite, des auteurs ont attaqué la vision canonique des Promessi sposi.

20. Parmi les textes récents qui ont attaqué cette vision, nous nous référons ici à A. Spranzi, Il segreto di Alessandro Manzoni..., op. cit., p. 205.

21. "come il Caccetta, che continuo a ritenere il vero spunto di due personaggi manzoniani, l'Innominato e Don Rodrigo", interview avec l'auteur in S. Vassalli, La chimera, Novara, Interlinea, 2003, p. 84.

22. Cf. Nesi, Sebastiano Vassalli, Fiesole, Cadmo, 2005. Le livre est approximatif quant à la sorcellerie: "Quasi tremila donne furono bruciate fra il 1560 e il $1670 »$. Dans cet extrait et dans la suite, l'auteur ne spécifie pas si cette donnée concerne la seule province de Novare ou l'Italie ou l'Europe entière. De plus, aucune référence bibliographique n'est offerte au lecteur.

23. La chasse aux sorcières fut un phénomène qui entraîna un nombre très vague de victimes entre XIV et XVIII' siècle (on parle d'un million, de cent milles et d'autres chiffres souvent hyperboliques qui d'après nous confondent procès et condamnations). En tout cas, quasiment toutes les exécutions eurent lieu dans les pays protestants. Les exécutions en Italie ont été peu nombreuses. Pourquoi la révélation de Vassalli, à quinze ans de distance, n'aurait-elle pas engendrée une étude anthropologique?

24. Une question intéressante est aussi celle du respect du genre littéraire, car, de ce point de vue, le fait qu'Antonia ait existé acquiert un poids considérable. Selon Manzoni, dans le roman historique, les personnages qui ont réellement existé doivent agir au second plan. Si Vassalli utilisait un personnage du plan historique en tant que protagoniste, il contredirait les règles fixées par Manzoni pour le genre. Si par contre Antonia est un personnage inventé, le nihilisme produit par son histoire s'oppose délibérément à la providence manzonienne. 